\title{
Management of concomitant mild to moderate functional mitral regurgitation during aortic valve surgery for severe aortic insufficiency
}

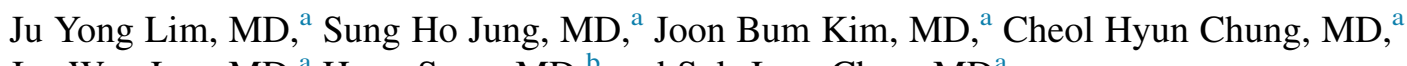
Jae Won Lee, MD, ${ }^{a}$ Hyun Song, MD, ${ }^{b}$ and Suk-Jung Choo, MD $^{\mathrm{a}}$

Objectives: The optimal management of mild to moderate functional mitral regurgitation (FMR) during aortic valve replacement (AVR) for severe aortic insufficiency (AI) is poorly defined. We aimed to investigate the fate of FMR after AVR with or without concomitant mitral annuloplasty (MAP) and to identify the risk factors and clinical implications of persistent FMR.

Methods: Between June 1996 and August 2011, 155 patients with mild to moderate FMR undergoing AVR for severe AI were reviewed. The preoperative MR grade was mild in 101 patients $(65 \%)$ and moderate in 54 patients $(35 \%)$. Persistent FMR was defined as MR grade remaining the same or increased on the last follow-up echocardiogram.

Results: The mean follow-up duration was $4.5 \pm 3.9$ years. FMR improved in $88 \%$ of the patients. On multivariate analysis, left ventricular end-diastolic dimension (LVEDD) reduction after AVR was identified as the only predictor for FMR improvement ( $P=.004$; hazard ratio, 0.927 ; confidence interval, 0.881 to 0.977 ). Concomitant MAP did not show additional benefit in preventing persistent FMR $(P=.35)$. Although no survival difference was observed between the patients with and without persistent FMR $(P=.78)$, persistent FMR was associated with greater heart failure events $(P<.001)$.

Conclusions: Mild to moderate FMR as a result of severe AI improved with AVR in most patients with or without concomitant MAP. Poor postoperative LVEDD reduction was the only risk factor for persistent FMR. Because persistent FMR tended to be associated with heart failure events, close echocardiographic monitoring and proactive medical management are recommended in patients showing poor LVEDD reduction after AVR. (J Thorac Cardiovasc Surg 2014;148:441-6)

Functional mitral regurgitation (FMR) is a common finding in aortic valve disease patients. ${ }^{1,2}$ However, adequate guidelines for its management during aortic valve surgery are lacking. ${ }^{3}$ Although previous studies have investigated the fate and clinical impact of mild to moderate FMR in patients undergoing aortic valve replacement (AVR), they were mostly conducted mostly in patients with aortic valve stenosis $^{4-8}$ and the literature addressing the fate of FMR specifically in the setting of severe aortic insufficiency (AI) has been limited. Even less is known about the outcome and the potential benefits of concomitant mitral annuloplasty. ${ }^{9}$ Therefore, we sought to investigate the following: (1) the fate of mild to moderate FMR after

\footnotetext{
From the Department of Thoracic and Cardiovascular Surgery, ${ }^{\mathrm{a}}$ Asan Medical Center, University of Ulsan, College of Medicine, Seoul, Korea; and Department of Thoracic and Cardiovascular Surgery, ${ }^{\mathrm{b}}$ The Catholic University of Korea, Seoul St. Mary Hospital, Seoul, Korea.

Disclosures: Authors have nothing to disclose with regard to commercial support.

Received for publication Jan 3, 2013; revisions received Aug 14, 2013; accepted for publication Sept 10, 2013; available ahead of print Nov 6, 2013.

Address for reprints: Suk-Jung Choo, MD, Asan Medical Center, University of Ulsan

College of Medicine, 388-1 Pungnap-Dong Songpa-Gu, Seoul 138-736,

South Korea (E-mail: sjchoo@amc.seoul.kr).

$0022-5223 / \$ 36.00$

Copyright (C) 2014 by The American Association for Thoracic Surgery

http://dx.doi.org/10.1016/j.jtcvs.2013.09.030
}

AVR for severe AI, (2) the effect of concomitant mitral annuloplasty (MAP) in the management of mild to moderate FMR, and (3) the risk factors and clinical implications of persistent FMR.

\section{PATIENTS AND METHODS \\ Patients}

Between June 1996 and August 2011, 779 patients underwent AVR for predominantly severe AI. Patients who had trivial $(1+)$ or severe $(4+)$ FMR, MR caused by structurally abnormal mitral valve and subvalvular apparatus such as rheumatic or degenerative pathology, and ischemic MR with regional wall motion abnormality were excluded. Based on this criteria, a total of 155 patients with predominantly severe AI with mild to moderate functional MR constituted the subject of the present study. Clinical characteristics and echocardiographic and surgical data were obtained retrospectively by reviewing the patients' medical records. The present study was approved by the Asan Institutional Review Board with waiver of individual patient consent.

\section{Echocardiographic FMR Grading and Follow-up Evaluation}

Transthoracic and transesophageal echocardiography were performed preoperatively in all of the patients to assess the etiology and severity of the MR. FMR was defined as central MR caused by tethering and fluttering in the absence of morphologic abnormalities of the mitral leaflets such as thickening or calcification. Any MR with regional left ventricular wall motion abnormality, suggesting an ischemic etiology, also was excluded. MR 


\section{Abbreviations and Acronyms \\ $\mathrm{AI} \quad=$ aortic insufficiency \\ AVR $=$ aortic valve replacement \\ $\mathrm{CHF}=$ congestive heart failure \\ FMR = functional mitral regurgitation \\ LVEDD $=$ left ventricular end-diastolic dimension \\ LVESD $=$ left ventricular end-systolic dimension \\ MAP = mitral annuloplasty}

grade was assessed by measuring the maximum color flow Doppler regurgitant jet area from the left ventricle to the left atrium during the systolic phase on a 4-chamber or 2-chamber view. The FMR was graded as none (0), trivial $(1+)$, mild $(2+)$, moderate $(3+)$, or severe $(4+)$ on an ordinal scale. All of the patients had a postoperative transthoracic echocardiogram before being discharged. Follow-up echocardiography was performed 6 months or 1 year after surgery in $88 \%$ of the patients. Biannual echocardiographic follow-up evaluation was recommended after the first year.

\section{Surgical Techniques}

Aortic valve replacement was performed by any 1 of 4 surgeons. Aortobicaval cannulation was used in a routine manner, with institution of antegrade and retrograde cardioplegia under mild hypothermia. All of the patients underwent AVR with either a mechanical or bioprosthetic valve as appropriate for the patient's age and clinical condition. Valve size was determined by intraoperative visual inspection of the aortic annulus and sizing of the left ventricular outflow tract. During this study period, there were no specific guidelines available for MAP. As a result, the decision to perform a concomitant MAP as well as choosing the type of mitral annuloplasty ring was left to the surgeon's discretion. The determination of ring size was based on the intercommissural distance and the anterior mitral valve leaflet height.

\section{Follow-up Evaluation}

Data collection was performed until October 2011 through interviews during regular outpatient clinic visits or by telephone inquiry. Follow-up evaluation was complete in $92 \%$ of the patients. Early mortality was defined as in-hospital death or death within 30 days of surgery. Mortality was categorized as cardiac or noncardiac based on the contents of the medical records. All deaths were considered to be of cardiac origin unless a noncardiac origin was established.

The primary study end point was cardiac death. The secondary end points included changes in the FMR grade after AVR and new-onset congestive heart failure (CHF) events (death or readmission caused by CHF) during the follow-up period. FMR was documented as either improved or persistent. Persistent FMR was defined as an MR grade that either remained the same or was aggravated in the last echocardiographic follow-up evaluation from the preoperative finding.

\section{Statistical Analysis}

Categoric variables are presented as frequencies and percentages, and continuous variables are expressed as the mean \pm standard deviation. Cumulative incidence rates of individual and composite outcomes were estimated by the Kaplan-Meier method and compared by the log-rank test. To reduce the impact of treatment selection bias and confounding potential in this observational study, we performed a rigorous adjustment for significant differences in patient characteristics by using Cox proportional-hazards regression models. Results were expressed as hazard ratios with $95 \%$ confidence intervals. All reported $P$ values are 2-sided, and $P$ values less than .05 were considered statistically significant. SAS software version 9.1 (SAS Institute, Inc, Cary, NC) was used for statistical analysis.

\section{RESULTS \\ Preoperative Characteristics and Surgical Data}

The preoperative clinical characteristics are shown in Table 1 . The mean age of the patients was $56 \pm 14$ years. Severe AI was the predominant aortic valve pathology in all of the patients, with only 2 patients showing mixed aortic valve disease. Grade 2+ FMR was observed in 101 patients $(65 \%)$ and grade $3+$ FMR was observed in 54 patients $(35 \%)$. The mean left ventricular end-systolic dimension (LVESD) and left ventricular end-diastolic dimension (LVEDD) were $50.5 \pm 11.0 \mathrm{~mm}$ and $69.5 \pm 9.4 \mathrm{~mm}$, respectively. MAP was performed in 22 patients (Table 2); preoperative FMR grade was $3+$ in 19 patients (86\%). The mean MAP ring size was $30.6 \pm 2.9 \mathrm{~mm}$.

\section{Clinical Outcomes}

There were 4 early deaths (2.6\%). Two of them occurred in patients who had received concomitant MAP for preoperative FMR grade $3+$. The mean follow-up duration was $53.5 \pm 47$ months. There were 22 late mortalities $(14.2 \%)$, of which 3 were caused by cancer-related complications (1 advanced gastric cancer and 2 lung cancers). Therefore, the overall cardiac-related mortality rate was $12.5 \%$. CHF events occurred in 4 patients. Two of the patients were rehospitalized with aggravated dyspnea and showed symptomatic improvement with medical management, but the other 2 patients who had very low postoperative left ventricular ejection fraction (ejection fraction, 16\%) died of CHF 4 and 9 years after AVR. There were 7 reoperations during the follow-up period. Most of the reoperations were for aortic valve-related problems such as endocarditis or paravalvular leakage.

\section{Changes in Echocardiographic Data After AVR}

Postoperative echocardiographic follow-up evaluation was performed over a mean follow-up duration of 24.3 \pm 11.8 months. The changes in the FMR during the follow-up period are shown in Figure 1. FMR improved after aortic valve surgery in $88 \%$ (133 of 151) of the patients and persisted in $12 \%$ (18 of 151 ). When comparing the FMR from the preoperative state, $84 \%$ (83 of 99) of the patients with grade $2+$ FMR and $96 \%$ (50 of 52) of the patients with grade $3+$ FMR showed improvement. The mean LVESD and LVEDD also improved from $50.5 \pm$ $11.0 \mathrm{~mm}$ and $69.5 \pm 9.4 \mathrm{~mm}$ to $36.3 \pm 10.4 \mathrm{~mm}$ and $52.4 \pm 8.6 \mathrm{~mm}$, respectively. Among the 20 surviving patients who underwent concomitant MAP, FMR persisted in 1 patient. 
TABLE 1. Patient characteristics

\begin{tabular}{|c|c|c|}
\hline Variables & Patients $(n=155)$ & $\%$ \\
\hline Age (y) & $56 \pm 14$ & \\
\hline Female sex & 50 & 32 \\
\hline Hypertension & 54 & 35 \\
\hline Diabetes & 7 & 4.5 \\
\hline Coronary artery disease & 17 & 11 \\
\hline Chronic renal failure & 3 & 2 \\
\hline Atrial fibrillation & 20 & 12.9 \\
\hline \multicolumn{3}{|l|}{ NYHA functional class } \\
\hline I & 40 & 25.8 \\
\hline II & 54 & 34.8 \\
\hline III & 45 & 29 \\
\hline IV & 16 & 10.3 \\
\hline $\operatorname{LVEF}(\%)$ & $50.2 \pm 12.5$ & \\
\hline $\operatorname{LVESD}(\mathrm{mm})$ & $50.5 \pm 11.0$ & \\
\hline LVEDD (mm) & $69.5 \pm 9.4$ & \\
\hline LA size (mm) & $47.3 \pm 5.1$ & \\
\hline RV systolic pressure (mm Hg) & $36.5 \pm 16.9$ & \\
\hline \multicolumn{3}{|l|}{ Etiology of AI } \\
\hline Degenerative & 41 & 26.4 \\
\hline Root dilatation & 34 & 21.9 \\
\hline Endocarditis & 34 & 21.9 \\
\hline Rheumatic & 3 & 1.9 \\
\hline Others & 43 & 27.7 \\
\hline \multicolumn{3}{|l|}{ TR grade } \\
\hline I & 75 & 48.4 \\
\hline II & 17 & 11 \\
\hline III & 12 & 7.7 \\
\hline IV & 1 & 0.6 \\
\hline \multicolumn{3}{|l|}{ FMR grade } \\
\hline II & 101 & 65.1 \\
\hline III & 54 & 34.8 \\
\hline
\end{tabular}

NYHA, New York Heart Association; $L V E F$, left ventricle ejection fraction; $L A$, left atrium; $R V$, right ventricle; $T R$, tricuspid regurgitation; $L V E S D$, left ventricular end-systolic dimension; $L V E D D$, left ventricular end-diastolic dimension; $A I$, aortic insufficiency; $F M R$, functional mitral regurgitation.

\section{Risk Analysis for Persistent FMR and Its Clinical Impact}

The patients were divided into 2 groups after AVR based on the FMR status of their last transthoracic echocardiography (ie, improved $[\mathrm{n}=133]$ and persistent

TABLE 2. Surgical data

\begin{tabular}{lc}
\hline \multicolumn{1}{c}{ Concomitant surgery } & $\mathbf{N}(\%)$ \\
\hline Mitral valve repair & $22(14)$ \\
Medtronics Duran* & 3 \\
St Jude Tailor $\dagger$ & 4 \\
Carpentier-Edwards Physio $\ddagger$ & 11 \\
Cosgrove-Edwards $\ddagger$ & 4 \\
Tricuspid valve repair & $12(8)$ \\
Maze surgery & $7(5)$ \\
Ascending aorta replacement & $10(6)$ \\
Coronary artery bypass surgery & $10(6)$ \\
\hline *Medtronics, Inc, Minneapolis, Minn. $\nmid$ St Jude Medical, Inc, St Paul, Minn. \\
$\ddagger$ Edwards Lifesciences, Irvine, Calif.
\end{tabular}

FMR [ $\mathrm{n}=18])$. Preoperative left ventricular ejection fraction was $50.56 \pm 12.8$ in the improved group and $49.17 \pm$ 9.3 in the persistent FMR group $(P=.58)$. On univariate analysis, poor reduction in LVESD, LVEDD, and the preoperative left atrium size; presence of chronic renal disease; and coronary artery disease were risk factors for persistent postoperative FMR. However, on multivariate analysis, only poor LVEDD reduction $(P=.004$; hazard ratio, 0.927; confidence interval $[C I], 0.881$ to 0.977 ) and preoperative left atrium size $(P=.017)$ were significant risk factors for persistent FMR (Table 3). The FMR type in $71 \%$ of the patients $(n=107)$ was type IIIb, and was type I in the remaining patients. No significant differences were observed in the incidence of persistent FMR between the 2 types of FMR. Concomitant MAP was not found to be of additional benefit in preventing persistent FMR. To exclude the influence of MAP from the evaluation, univariate and multivariate analyses were performed in the patients without MAP, but the results remained similar to that of the total patient cohort (Table 4).

Persistent FMR did not seem to affect the estimated long-term survival $(P=.709)$ (Figure 2). However, it is remarkable that all of the CHF events $(\mathrm{n}=4)$ occurred in the persistent postoperative FMR patients $(P<.001)$.

\section{DISCUSSION}

The present study was focused on assessing the course of mild to moderate FMR and the clinical impact of persistent postoperative FMR in patients undergoing AVR for predominantly severe AI. The results showed FMR improvement in most of the patients with AVR only. However, some patients showed persistent FMR, which had an adverse impact on the clinical outcomes, especially with respect to a greater incidence of CHF-related events. The most important factor relating to persistent FMR after AVR was poorly reduced LVEDD. Concomitant MAP was not necessarily found to be of greater benefit in preventing persistent FMR.

Although FMR associated with aortic valve disease is relatively common, its course may vary depending on the predominant aortic valve pathology (ie, stenosis or regurgitation). Although numerous studies were conducted to define the relationship between FMR and aortic valve disease, the predominant aortic valve pathology in most of these studies was aortic stenosis, ${ }^{6,8,10,11}$ with only a few of them dealing with predominantly severe AI. Even in the studies that purportedly investigated mixed aortic pathologies, the proportion of AI patients was small and the distinction between the 2 pathologies was unclear. ${ }^{7,12}$ It is our contention that distinguishing the FMR in relation to the type of aortic valve pathology is important because the underlying left ventricular remodeling behavior may differ accordingly, and thereby affect the course of the FMR and consequently the overall clinical 


\section{Preoperative FMR grade Postoperative FMR grade}
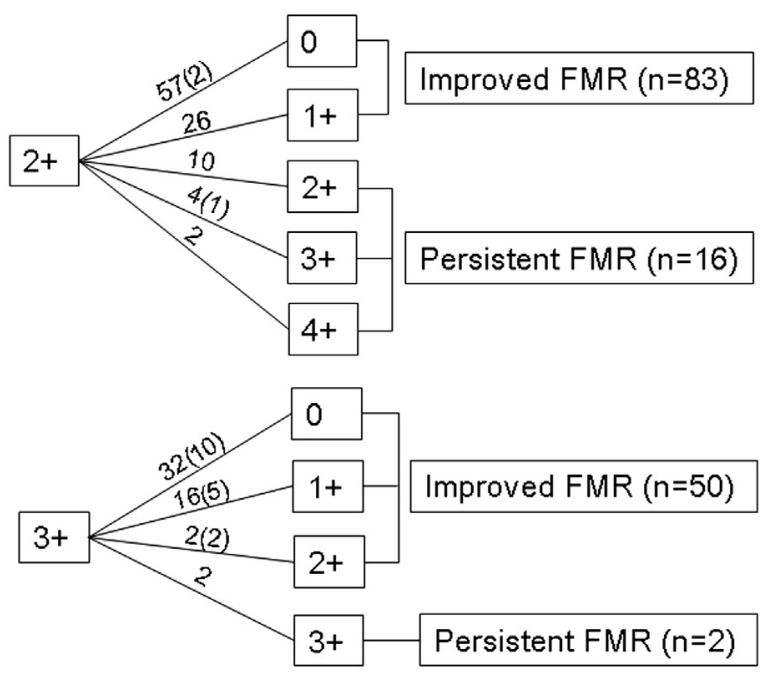

() - number of patients who had concomitant mitral annuloplasty

FIGURE 1. The changes in the degree of FMR after AVR. FMR, Functional mitral regurgitation; $A V R$, aortic valve replacement.

outcome. The left ventricular remodeling in aortic stenosis is characterized by ventricular hypertrophy followed by dilatation as a delayed response to disease progression. On the other hand, the left ventricular remodeling response to severe $\mathrm{AI}$ is usually dilatation and increase in

TABLE 3. Cox proportional hazards model analyzing predictors for persistent FMR in all patients $(\mathbf{n}=\mathbf{1 5 1})$

\begin{tabular}{lrrrr}
\hline \multicolumn{1}{c}{ Variables } & $\begin{array}{c}\text { Hazard } \\
\text { ratio }\end{array}$ & $\begin{array}{c}\text { 95\% confidence } \\
\text { interval }\end{array}$ & $\boldsymbol{P}$ value \\
\hline Univariate analysis & & & & \\
Age & 1.003 & 0.969 & 1.038 & .859 \\
Sex & 1.531 & 0.588 & 3.987 & .383 \\
Hypertension & 0.455 & 0.100 & 2.068 & .308 \\
Diabetes & 2.699 & 0.339 & 21.467 & .348 \\
NYHA & 0.981 & 0.365 & 2.636 & .969 \\
Coronary artery disease & 3.352 & 1.178 & 9.543 & $.023^{*}$ \\
Dialysis & 10.703 & 1.237 & 92.623 & $.031^{*}$ \\
Atrial fibrillation & 2.333 & 0.748 & 7.275 & .144 \\
LVESD difference & 0.944 & 0.897 & 0.993 & $.026^{*}$ \\
LVEDD difference & 0.923 & 0.877 & 0.972 & $.002^{*}$ \\
LA size & 1.074 & 1.000 & 1.153 & .051 \\
RV pressure & 0.977 & 0.943 & 1.012 & .189 \\
Tricuspid regurgitation & 1.870 & 0.525 & 6.660 & .334 \\
Mitral annuloplasty & 0.381 & 0.050 & 2.884 & .350 \\
LVEF & 0.974 & 0.938 & 1.011 & .167 \\
Multivariate analysis & & & & \\
LVEDD difference & 0.927 & 0.881 & 0.977 & $.004 *$ \\
\hline
\end{tabular}

NYHA, New York Heart Association; LVESD difference, preoperative LVESD value postoperative LVESD value; $L V E D D$ difference, preoperative LVEDD value - postoperative LVEDD value; LVESD, left ventricular end-systolic dimension; $L V E D D$, left ventricular end-diastolic dimension; $L A$, left atrium; $R V$, right ventricle; $L V E F$, left ventricle ejection fraction. $* P<.05$.
TABLE 4. Cox proportional hazards model for predictors of persistent FMR in patients without concomitant mitral annuloplasty $(\mathbf{n}=\mathbf{1 3 1})$

\begin{tabular}{lccrc}
\hline \multicolumn{1}{c}{ Variables } & $\begin{array}{c}\text { Hazard } \\
\text { ratio }\end{array}$ & \multicolumn{2}{c}{$\begin{array}{c}\mathbf{9 5} \% \text { confidence } \\
\text { interval }\end{array}$} & P value \\
\hline Univariate analysis & & & & \\
Age & 1.004 & 0.969 & 1.041 & .807 \\
Sex & 1.466 & 0.544 & 3.945 & .449 \\
Hypertension & 0.221 & 0.028 & 1.717 & .149 \\
Diabetes & 2.329 & 0.293 & 18.521 & .424 \\
NYHA & 1.239 & 0.452 & 3.397 & .677 \\
Coronary artery disease & 3.963 & 1.249 & 12.578 & $.019^{*}$ \\
Dialysis & 9.291 & 1.074 & 80.385 & $.043^{*}$ \\
Atrial fibrillation & 2.240 & 0.630 & 7.964 & .213 \\
LVESD difference & 0.953 & 0.903 & 1.006 & .079 \\
LVEDD difference & 0.928 & 0.875 & 0.984 & $.013^{*}$ \\
LA size & 1.136 & 1.041 & 1.239 & $.004^{*}$ \\
RV pressure & 0.983 & 0.950 & 1.017 & .315 \\
Tricuspid regurgitation & 2.407 & 0.654 & 8.857 & .186 \\
LVEF & 0.968 & 0.930 & 1.007 & .106 \\
Multivariate analysis & & & & \\
LVEDD difference & 0.927 & 0.749 & 0.977 & $.004^{*}$ \\
LA size & 1.121 & 1.020 & 1.231 & $.017^{*}$ \\
\hline
\end{tabular}

NYHA, New York Heart Association; LVESD difference, preoperative LVESD value postoperative LVESD value; $L V E D D$ difference, preoperative LVEDD value - postoperative LVEDD value; $L A$, left atrium; $R V$, right ventricle; $L V E F$, left ventricle ejection fraction; $L V E S D$, left ventricular end-systolic dimension; $L V E D D$, left ventricular end-diastolic dimension. ${ }^{*} P<.05$

LV mass. Therefore, the onset and LV dysfunctional state secondary to myocardial remodeling in patients with predominantly severe AI may be qualitatively different from aortic stenosis. From this perspective, the present

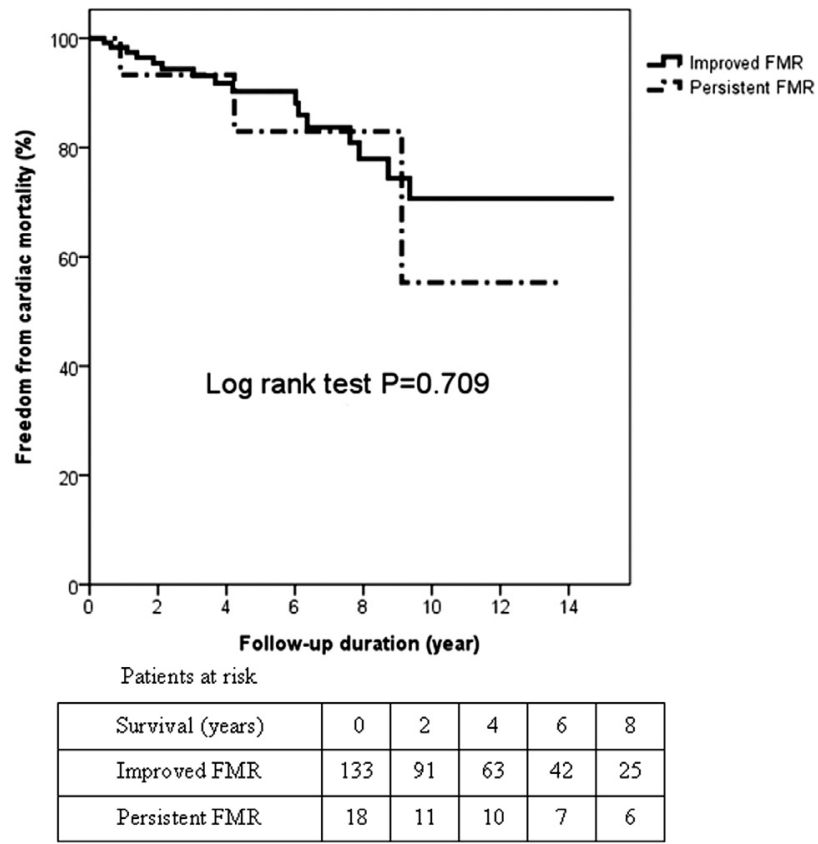

FIGURE 2. Freedom from cardiac mortality. FMR, Functional mitral regurgitation. 
study was remarkable in having focused specifically on LV remodeling and FMR in AVR patients with predominantly severe AI. The reversal of FMR after AVR in these patients was rapid with significant reverse remodeling that was characterized by decreased LVEDD and FMR improvement within the first few months of surgery. Although the findings of this study may guide proactive postoperative medical treatment in those patients showing poor LVEDD reduction, the surgical utility remains limited because significant preoperative factors predicting persistent postoperative FMR were not identified.

Until recently, most studies on FMR involved patients with MR ranging between $1+$ or $2+.^{7,9,12,13}$ In this study, we chose to limit our patients to those with MR grade $2+$ or $3+$ MR because the treatment of FMR in this group of patients remains the most controversial. A total of $35 \%$ of the patients had grade $3+$ FMR and, remarkably, most of them $(96 \%)$ showed improvement in their MR after AVR alone without concomitant MAP. Therefore, the results seemed to suggest that AVR alone may be sufficient to induce the necessary reverse remodeling for correcting the mild to moderate FMR, but the relatively small population of 155 patients in the present study warrants further investigation over a larger cohort and longer duration to adequately assess the clinical implications.

Although it is intuitive to expect a greater preventive effect on persistent postoperative FMR by concomitant MAP, multivariate analysis showed no additional benefit with MAP, even in patients with preoperative grade $3+$ MR. These findings corroborate the views argued by Wan et $\mathrm{al}^{9}$ that AVR alone in most cases is highly effective in resolving mild to moderate FMR at the time of AVR. In a review by Abdullah et al, ${ }^{5}$ the cause of MR worsening after AVR was suggested to be multifactorial. ${ }^{10,12,14-16}$ In the present study, poor reduction in LVEDD was the only significant risk factor identified for persistent FMR after AVR $(P=.004)$. Joo et $\mathrm{al}^{7}$ also suggested LVEDD increase to be predictive of persistent FMR on univariate analysis. These findings stress the significant role of adverse LV remodeling in contributing to persistent FMR after AVR. Although the size of the preoperative LVEDD was not a significant predictor of persistent FMR, changes in LVEDD (ie, poorly reduced LVEDD after AVR) were identified as having an adverse influence on the clinical outcomes, verifying the importance of the postoperative LV remodeling behavior. The clinical impact of persistent FMR was most readily apparent through its greater association with increased $\mathrm{CHF}$ events during the follow-up period. This was consistent with the reported long-term outcomes of previous studies that showed an increased incidence of CHFrelated events in patients with persistent FMR. ${ }^{6,12}$ The tendency for a decrease in the LVEDD to be associated with greater FMR improvement as observed in the present study is consistent with the outcomes reported by Ruel et al. ${ }^{12}$

\section{Study Limitations}

Limitations include the retrospective nature of the present study and the possibility of treatment bias by the attending surgeons. The relatively short follow-up duration and the small number of CHF events are further limitations. Although the relatively homogenous study population consisting of patients with predominantly severe AI adds support to the arguments advocated in the present study, further long-term studies in a larger study cohort are warranted to identify the preoperative risk factors of persistent postoperative FMR and the surgical utility of the present study findings.

\section{CONCLUSIONS}

Mild to moderate FMR associated with severe AI showed improvement after aortic valve surgery alone in most patients. Concomitant MAP did not result in an additional beneficial effect in preventing persistent FMR. The only risk factor for persistent FMR after AVR was poor LVEDD reduction on follow-up echocardiography. Although persistent FMR did not affect the overall survival rate, it tended to be associated with a higher incidence of congestive heart failure events during the follow-up period. Therefore, proactive medical management is recommended in patients with poor LVEDD reduction after AVR.

\section{References}

1. Moazami N, Diodato MD, Moon MR, Lawton JS, Pasque MK, Herren RL, et al. Does functional mitral regurgitation improve with isolated aortic valve replacement? J Card Surg. 2004;19:444-8.

2. Sabbah HN, Rosman H, Kono T, Alam M, Khaja F, Goldstein S. On the mechanism of functional mitral regurgitation. Am J Cardiol. 1993;72: 1074-6.

3. Bonow RO, Carabello BA, Chatterjee K, de Leon AC Jr, Faxon DP, Freed MD, et al. 2008 focused update incorporated into the ACC/AHA 2006 guidelines for the management of patients with valvular heart disease - a report of the American College of Cardiology American Heart Association task force on practice guidelines (writing committee to revise the 1998 guidelines for the management of patients with valvular heart disease). Endorsed by the Society of Cardiovascular Anesthesiologists, Society for Cardiovascular Angiography and Interventions, and Society of Thoracic Surgeons. J Am Coll Cardiol. 2008; 52:E1-142.

4. Absil B, Dagenais F, Mathieu P, Métras J, Perron J, Baillot R, et al. Does moderate mitral regurgitation impact early or mid-term clinical outcome in patients undergoing isolated aortic valve replacement for aortic stenosis? Eur J Cardiothorac Surg. 2003;24:217-22.

5. Alghamdi AA, Elmistekawy EM, Singh SK, Latter DA. Is concomitant surgery for moderate functional mitral regurgitation indicated during aortic valve replacement for aortic stenosis? A systematic review and evidence-based recommendations. J Card Surg. 2010;25:182-7.

6. Jeong DS, Park PW, Sung K, Kim WS, Yang JH, Jun TG, et al. Long-term clinical impact of functional mitral regurgitation after aortic valve replacement Ann Thorac Surg. 2011;92:1339-45.

7. Joo HC, Chang BC, Cho SH, Youn YN, Yoo KJ, Lee S. Fate of functional mitral regurgitation and predictors of persistent mitral regurgitation after isolated aortic valve replacement. Ann Thorac Surg. 2011;92:82-7. 
8. Waisbren EC, Stevens LM, Avery EG, Picard MH, Vlahakes GJ, Agnihotri AK. Changes in mitral regurgitation after replacement of the stenotic aortic valve. Ann Thorac Surg. 2008;86:56-62.

9. Wan CK, Suri RM, Li Z, Orszulak TA, Daly RC, Schaff HV, et al. Management of moderate functional mitral regurgitation at the time of aortic valve replacement: is concomitant mitral valve repair necessary? J Thorac Cardiovasc Surg. 2009; 137:635-640.e1.

10. Brasch AV, Khan SS, DeRobertis MA, Kong JH, Chiu J, Siegel RJ. Change in mitral regurgitation severity after aortic valve replacement for aortic stenosis. Am J Cardiol. 2000;85:1271-4.

11. Vanden Eynden F, Bouchard D, El-Hamamsy I, Butnaru A, Demers P, Carrier M, et al. Effect of aortic valve replacement for aortic stenosis on severity of mitral regurgitation. Ann Thorac Surg. 2007;83:1279-84.

12. Ruel M, Kapila V, Price J, Kulik A, Burwash IG, Mesana TG. Natural history and predictors of outcome in patients with concomitant functional mitral regurgitation at the time of aortic valve replacement. Circulation. 2006;114: I541-6.

13. Goland S, Loutaty G, Arditi A, Snir E, Abend I, Caspi A. Improvement in mitral regurgitation after aortic valve replacement. Isr Med Assoc J. 2003;5: $12-4$.

14. Christenson JT, Jordan B, Bloch A, Schmuziger M. Should a regurgitant mitral valve be replaced simultaneously with a stenotic aortic valve? Tex Heart Inst J. 2000;27:350-5.

15. Harris KM, Malenka DJ, Haney MF, Jayne JE, Hettleman B, Plehn JF, et al. Improvement in mitral regurgitation after aortic valve replacement. Am J Cardiol. 1997;80:741-5.

16. Tassan-Mangina S, Metz D, Nazeyllas P, Torossian F, Pop C, Bertrand J, et al. Factors determining early improvement in mitral regurgitation after aortic valve replacement for aortic valve stenosis: a transthoracic and transesophageal prospective study. Clin Cardiol. 2003;26:127-31. 